\title{
TAGUNG
}

\section{Vielfalt ohne Einheit}

\author{
Frédéric Krumbein*
}

Eckart D. Stratenschulte gab einen Überblick über die verschiedenen Integrationsstrukturen in Europa. Zuerst definierte er Integration: Integration meine eine auf Dauer angelegte Kooperation mehrerer Staaten, die gemeinsame Ziele verfolgten und einen Teil der eigenen Souveränität transferierten. Ein vollständiger Souveränitätstransfer oder kein Transfer von Souveränität falle nicht unter den Integrationsbegriff.

Die verschiedenen Integrationsstrukturen in Europa ließen sich nach fünf Kriterien unterscheiden: Ziele, geografische Reichweite, Integrationstiefe, Verbindlichkeit sowie Integrationskapazität, das heißt die reale Umsetzung der formalen Integrationsstrukturen.

Die größte Organisation in Europa sei die Organisation für Sicherheit und Zusammenarbeit in Europa (OSZE), die alle Staaten in Europa sowie die USA und Kanada umfasse. Insgesamt seien 57 Staaten Mitglied in dieser Organisation. Die Hauptaufgabe der OSZE, ursprünglich als Konferenz für Sicherheit und Zusammenarbeit bezeichnet, stellte die Moderation des Ost-West-Konflikts dar. Seit seinem Ende liege ein Fokus ihrer Arbeit auf der Wahlbeobachtung und der Förderung der Demokratie. Allerdings sei die Verbindlichkeit der Ziele sehr gering. Entsprechend könne die OSZE in erster Linie als ein Forum für Debatten betrachtet werden. Die einzigen Staaten, die keine Mitglieder sind, seien die ,de-facto-Staaten', wie beispielsweise der Kosovo.

Der Europarat könne als die zweitgrößte Organisation betrachtet werden. Er habe 47 Mit-

\section{Heilsame Vielfalt? Integrationsstrukturen in Europa}

\author{
13.-15. Januar 2014, Berlin
}

Tagung des Arbeitskreises Europäische Integration und der Europäischen Akademie Berlin

Mit freundlicher Unterstützung der Europäischen Kommission

Chaos oder Vielfalt? Integrationsstrukturen im Europa des 21. Jahrhunderts im Überblick Prof. Dr. Eckart D. STRATENSCHULTE, Europäische Akademie Berlin

Die „Eurasische Union“: Reintegration des postsowjetischen Raums oder Teil einer „Gemeinschaft von Lissabon bis Wladiwostock"?

Prof. Dr. Hannes ADOMEIT, Colleg of Europe, Brügge

Entscheidungszentrum Eurozone: Die Marginalisierung der Union?

Prof. Dr. Andreas MAURER, Universität Innsbruck

Heute Euroland - morgen EU? Montenegro und seine europäische Währung

Prof. Dr. Radoje ŽUGIĆ, Finanzminister Montenegro, Podgorica

Gibraltar und die EU: Die Konsequenzen des

Konflikts zwischen Spanien und

Großbritannien für Gibraltar und den

Europäischen Integrationsprozess

Prof. Dr. Alejandro DEL VALLE GÁLVEZ, University of Cadiz

Ein Staat - in Gottes Namen: Der Vatikanstaat

Dr. Paul WIDMER, Schweizer Botschafter für den Heiligen Stuhl, Bern

* Dr. Frédéric Krumbein, Geschäftsführer des Arbeitskreises Europäische Integration, Berlin. 
glieder und inzwischen über zweihundert Konventionen verabschiedet. Mit Ausnahme der Europäischen Menschenrechtskonvention (EMRK) und ihrer Zusatzprotokolle sei keine Konvention von allen Mitgliedern ratifiziert worden. Die Verbindlichkeit ihrer Ziele, die Förderung von Demokratie und Menschenrechten, könne als eher gering eingestuft werden. Die einzige Ausnahme bilde der Europäische Gerichtshof für Menschenrechte, der auf Basis der EMRK für die Europaratsmitglieder bindende Urteile fälle.

Die Europäische Union verfüge über weniger Mitglieder als der Europarat, habe aber eine deutlich größere Verbindlichkeit und Integrationstiefe. Wenn man die verschiedenen Instrumente der Nachbarschafts- und Erweiterungspolitik miteinbeziehe, dann überlappten sich die Mitglieder des Europarates fast vollständig mit den Staaten, die an den verschiedenen EU-Instrumenten teilnähmen. Einzig Russland verfüge über einen Sonderstatus.

Die NATO ${ }^{1}$ verfolge seit ihrer Gründung die drei Ziele der Einhegung und Abwehr eines potenziellen Angriffs Russlands, der Integration der USA in die europäische Sicherheitsarchitektur sowie der Einbindung und „Zähmung" Deutschlands.

Benelux und die Nordische Partnerschaft könnten schließlich als Integrationsstrukturen betrachtet werden, die sich durch wenige Mitglieder, aber eine hohe Integrationstiefe auszeichneten.

Die Integrationsstrukturen in Osteuropa unterschieden sich deutlich von denen in West- und Gesamteuropa, da in den osteuropäischen Integrationsstrukturen das Problem in der Dominanz Russlands bestehe. Die Gemeinschaft Unabhängiger Staaten spiele inzwischen keine wichtige Rolle mehr. Als Nachfolger plane Russland nun eine Eurasische Union, welche

\author{
Ausgetreten und doch drin? Der Status von \\ Grönland im Hinblick auf die Europäische \\ Union \\ Dr. Damien DEGEORGES, Arctic Policy and Eco- \\ nomic Forum, Paris \\ Der Status Spitzbergens und die Konsequenzen \\ für die internationale Politik \\ Dr. Kristian ÅTLAND, Norwegisches Forsvarets \\ Froskningsinstitutt, Kjeller \\ Die de-facto- Staaten in Europa: Ärgernis oder \\ Vorboten der Veränderung \\ Prof. Dr. Otto LUCHTERHANDT, Universität \\ Hamburg \\ Der Europäische Wirtschaftsraum - \\ Mitgliedschaft minus in der EU? \\ Dr. Barbara LIPPERT, Stiftung Wissenschaft und \\ Politik, Berlin
}

die bestehende Zollunion zwischen Russland, Kasachstan und Belarus weiter vertiefen solle. Viele Experten stuften das Integrationspotenzial aber als gering ein (,Leuchtturm für die Gestrandeten"), selbst unter Einbeziehung der Staaten Armenien und Kirgisistan. Die Zukunft der Eurasischen Union werde von der Einbeziehung der Ukraine abhängen. Sollte sich die Ukraine stattdessen an der Europäischen Union orientieren, werde die Eurasische Union keine große Bedeutung erlangen.

Zusätzlich gebe es die Organisation für kollektive Sicherheit, die Russland, Armenien, Belarus, Kasachstan, Kirgisistan und Tadschikistan umfasse und formal kollektive Sicherheit garantiere. In der Praxis sei die Verbindlichkeit geringer als bei der Eurasischen Union einzustufen.

Insgesamt existiere trotz der vielen Organisationen kein Chaos in Europa, sondern die verschiedenen Integrationsstrukturen verfügten alle über eine eigene Bedeutung. Allerdings gebe es eine gewisse Konkurrenz zwischen einigen Integrationsstrukturen, wie den von

1 North Atlantic Treaty Organization. 
Russland dominierten osteuropäischen Organisationen und den von westlichen Staaten dominierten Organisationen.

Führt die Integration von Wenigen zur Desintegration der Vielen?

Andreas Maurer referierte über die Gefahr der Marginalisierung der Europäischen Union durch die Eurozone. Die Rechtsetzung der Europäischen Union in den Bereichen Wirtschaft und Währung habe seit dem Vertrag von Maastricht rapide zugenommen. Inzwischen würden pro Jahr etwa 90 bis 100 Rechtsakte in diesem Bereich verabschiedet. Insgesamt gebe es etwa 1.500 Rechtsakte, die von der Europäischen Union jedes Jahr verabschiedet werden. Die vielen verabschiedeten Rechtsakte in anderen Bereichen als der Wirtschafts- und Währungsunion zeigten die Bedeutung der Europäischen Union für zahlreiche Politikfelder, wie beispielsweise in der Agrar- oder der Außenpolitik.

Wenn man die Integrationsstrukturen betrachte, könne man eine zunehmende Vertiefung feststellen, ausgehend von der locker verfassten Mittelmeerunion, über die Zollunion mit der Türkei, den Europäischen Wirtschaftsraum, der Europäischen Union bis hin zur Eurogruppe. Die Mitgliedschaft in der Eurozone betreffe potenziell alle EU-Mitgliedstaaten mit Ausnahme Großbritanniens und Dänemarks. Alle anderen Staaten müssten, nach Erfüllung der entsprechenden Kriterien, Mitglied der Eurozone werden. Die Währung der Europäischen Union sei, laut den EU-Verträgen, der Euro. Dies würden alle EU-Mitglieder anerkennen, auch Großbritannien und Dänemark.

In der Europäischen Union zeigten sich zwei unterschiedliche Methoden der Kooperation von EU-Staaten. Erstens, es gebe eine verstärkte Zusammenarbeit, bei der einige Staaten enger zusammenarbeiteten, aber bei der alle Staaten die Ziele der Zusammenarbeit teilten. Es gebe also ,Opt-outs‘ im Rahmen der Strukturen der Europäischen Union. Dann gebe es faktische ,Opt-outs', wie beispielsweise die
Weigerung Deutschlands, die Vorratsdatenspeicherung als geltendes EU-Recht umzusetzen. Die einzige Institution, in der ausschließlich Mitglieder der Eurozone mitwirkten, sei die Europäische Zentralbank. Formal träfen die Staaten der Eurozone keine alleinigen Entscheidungen innerhalb der Europäischen Union. Allerdings verfügten die Staaten der Eurozone über eine Sperrminorität in den Entscheidungsgremien der Europäischen Union. Es gebe aber keine Evidenz dafür, dass die Eurostaaten diese Macht ausnutzen.

Zweitens, die Staaten der Eurozone würden zum Teil eigene Strukturen für die Eurozone schaffen und dadurch die Institutionen der Europäischen Union schwächen. So gebe es zum Beispiel Diskussionen über ein eigenes Parlament nur für die Eurozone. Diese Entwicklung habe eine neue Qualität. Es gebe aus Sicht von Maurer aber keinen Grund für ein eigenes Parlament oder eine Spaltung des bestehenden Europäischen Parlaments. Sollte man dies tun, so müsste das Europäische Parlament für eine Vielzahl von Politikbereichen differenzierte Strukturen schaffen. Innerhalb des Europäischen Parlaments herrsche breiter Konsens, eine solche Spaltung abzulehnen.

Letztlich gebe es nur eine Möglichkeit geltendes EU-Recht und die Nutzung der EU-Organe zu garantieren: Dies sei der Weg über den formalen europäischen Gesetzgebungsprozess, bei dem alle EU-Mitgliedstaaten sich beteiligten. Ohne die Beschreitung dieses Weges könnten Mitgliedstaaten zwar Abkommen und Verträge außerhalb der Europäischen Union schließen, wie den Fiskalpakt, aber die EUOrgane könnten nicht genutzt werden. Im Falle des Fiskalpaktes hätte es auch die Möglichkeit gegeben, eine Regierungskonferenz einzuberufen und auf diese Weise zu versuchen, Großbritannien durch Zugeständnisse zu überzeugen und den Fiskalpakt im Rahmen der Europäischen Union zu verabschieden. Aber Deutschland habe vermutlich die Intention verfolgt, die EU-Kommission und das Europäische Parlament zu schwächen und eine von den Regierungen der Eurozone dominierte neue Institution zu schaffen. 
In der Summe habe die Eurozone, insbesondere während der Finanz- und Schuldenkrise, einen Keil in die Europäische Union getrieben. Dieser Keil bestünde aber nicht so sehr zwischen einer Spaltung der Eurozone und dem Rest der Europäischen Union, sondern mehr in einer Spaltung der Eurozone in Nord- und Südstaaten.

\section{Montenegro - EU-Währung ohne EU-Mit- gliedschaft}

Radoye Žugić sprach über den Euro in Montenegro. Montenegro habe den Euro als Währung ohne gleichzeitig ein Mitglied der Europäischen Union zu sein. Normalerweise beschreite ein Staat den umgekehrten Weg und werde zuerst Mitglied der Europäischen Union und übernehme dann den Euro. Montenegro verwende seit mehr als zehn Jahren den Euro als Währung. Das Land strebe eine vollständige Integration in die Europäische Union und die NATO an. Žugić rechnete mit einer Erfüllung der EU-Beitrittskriterien innerhalb von sechs bis sieben Jahren.

Die Einführung einer Fremdwährung, zuerst der Deutschen Mark und später des Euro, erfolgte, um die grassierende Inflation in Montenegro mit der einheimischen Währung Dinar einzudämmen. Bei der Einführung des Euro habe Montenegro mit einer ausländischen Bank zusammengearbeitet, die Montenegro den Euro gegen Dinar verkauft habe. Die Einführung sei sowohl physisch, also mit Scheinen und Münzen, als auch für den bargeldlosen Zahlungstransfer erfolgt.

Die wirtschaftliche Entwicklung Montenegros habe wesentlich mit der Einführung des Euro begonnen. In den Jahren 2006 bis 2009 habe es einen wirtschaftlichen Boom mit Wachstumsraten von 8 bis 9 Prozent gegeben. Anschließend sei eine Rezession eingetreten. Erst seit 2013 wachse die Wirtschaft wieder. Seit der Einführung des Euro könne man die Inflation in Montenegro mit derjenigen der Eurozone vergleichen. Die Einführung des Euro habe außerdem dazu geführt, dass die ausländischen
Investitionen in Montenegro sich erhöht hätten. Die meisten Investitionen stammen aus Italien. Darüber hinaus gebe es nach Ansicht von Žugić keine negativen Auswirkungen auf die Eurozone, da die Menge der sich in Umlauf befindlichen Euro in Montenegro nur gering sei und die gesamte Geldmenge des Euro kaum beeinflusse. Montenegro wünsche sich eine Beibehaltung des Euro als Währung und werbe für dieses Ziel bei den Staaten der Eurozone.

\section{Der Vatikan - mehr Diplomaten als Einwoh- ner?}

Die Außenpolitik des Heiligen Stuhls war der Gegenstand des Vortrags von Paul Widmer. Zu Beginn seiner Ausführungen erläuterte er den Unterschied zwischen dem Staat Vatikanstadt als dem Träger staatlicher Macht mit einem Territorium von 44 Hektar und einer Bevölkerung von circa 600 Personen, der von einer Verwaltung (,Governatorato") geleitet wird, und dem Heiligen Stuhl, der die Führung der katholischen Kirche verkörpere. Der Heilige Stuhl sei seit alters her als Völkerrechtssubjekt sui generis anerkannt. Beide Völkerrechtssubjekte würden durch dieselbe Person, den Papst, verkörpert. In den auswärtigen Beziehungen habe die internationale Gemeinschaft es immer mit dem Heiligen Stuhl zu tun, bei dem auch die ausländischen Botschaften akkreditiert seien. Sofern eine Angelegenheit in erster Linie den Vatikanstaat betreffe, wie beispielsweise der Beitritt zur Anti-Folterkonvention der Vereinten Nationen, aber auch Währungs- und Zollverträge, handele der Heilige Stuhl im Namen des Vatikanstaats.

Der Heilige Stuhl unterhalte diplomatische Beziehungen zu 180 Staaten und verfüge über ein gut ausgebautes diplomatisches Netz. Er unterhalte 99 apostolische Nuntiaturen (Botschaften), nicht nur bei Staaten, sondern auch bei internationalen Organisationen wie den Vereinten Nationen, der OSZE, der Arabischen Liga oder der Europäischen Union. Der Nuntius sei auch und in erster Linie für die Verbindungen der jeweiligen nationalen katholischen Kirche mit dem Heiligen Stuhl zu- 
ständig. Auch dadurch verfüge der Heilige Stuhl über ein hervorragendes Netzwerk und sehr viele Informationen, die er auch bei zahlreichen Gesprächen mit führenden Politikern im Vatikan sammele. Fast jede Woche finde ein Staatsbesuch im Vatikan statt, bei dem der Papst sich mit einem Staatsoberhaupt austausche.

Die Außenpolitik des Heiligen Stuhls sei langfristig angelegt und orientiere sich an den Werten der katholischen Kirche sowie am Grundsatz der Neutralität. Der Heilige Stuhl sei nicht grundsätzlich pazifistisch, billige militärische Eingriffe jedoch nur als Ultima Ratio und zur Abwehr einer Aggression. Präventivkriege lehne er grundsätzlich ab. Von Papst Franziskus seien Veränderungen im Hinblick auf eine stärkere Orientierung auf die Bedürfnisse der Entwicklungsländer zu erwarten. Allerdings dürfe man auch die Veränderungen im Auftreten von Papst Franziskus im Vergleich zu seinen Vorgängern nicht überschätzen. Das starke institutionelle Gefüge der Kirche lasse nur eine langsame Entwicklung $\mathrm{zu}$ - und es sei auch nicht garantiert, dass diese stattfinden werde.

\section{Gibraltar - die letzte Kolonie in Europa?}

Alejandro del Valle Gálvez erläuterte den Konflikt um Gibraltar, der heute noch zwischen Spanien und Großbritannien bestehe. Durch den Vertrag von Utrecht sei Gibraltar 1713 an Großbritannien übertragen worden. Spanien sehe Gibraltar nach wie vor als Kolonie an und fordere die Entkolonialisierung, die es mit einer Rückgabe Gibraltars an Spanien gleichsetze. Der Vertrag von Utrecht schreibe vor, dass für den Fall, dass Großbritannien die Souveränität über Gibraltar abgebe, diese Spanien zufalle oder zumindest Spanien angeboten werden müsse. Mittlerweile sei eine partielle Souveränitätsübergabe erfolgt, allerdings an die Bevölkerung von Gibraltar - ein Fall, der 1713 nicht vorausgesehen worden sei.

Weitere Konflikte bestünden über die Rechte der beiden Staaten an den Gewässern um Gibraltar, die ursprüngliche und inzwischen weit- gehend von Großbritannien vereinnahmte neutrale Zone zwischen dem Felsen und dem spanischen Festland sowie die Landgewinnung an der Küste von Gibraltar. Die Bürger von Gibraltar können, auf der Basis eines Urteils des Europäischen Gerichtshofs für Menschenrechte in Straßburg, ihre Stimme zu den Wahlen zum Europäischen Parlament abgeben - als Teil des britischen Elektorats.

Im Jahr 2013 hätten sich die Auseinandersetzungen zwischen beiden Staaten durch einige Vorfälle, wie das Aufbringen spanischer Fischerboote durch die Polizei von Gibraltar, zugespitzt. Allerdings würden Spanien und Großbritannien die Organisationen, denen sie gemeinsam angehören, wie Europäische Union und NATO, mit diesem Konflikt nicht belasten. Lediglich in den Vereinten Nationen würden die unterschiedlichen Auffassungen immer wieder vorgebracht. Für Spanien stelle die ,Rückgewinnung' Gibraltars eine Frage der nationalen Ehre dar. Großbritannien wolle wiederum keinen Präzedenzfall schaffen, orientiere sich am Votum der Bevölkerung und wolle ebenfalls seine militärischen Anlagen auf dem Felsen nicht aufgeben.

\section{Grönland-europäische Bürger im ewigen Eis}

Damien Degeorges stellte die spezifische Situation Grönlands vor. Grönland sei ein selbstverwaltetes Gebiet, das zu Dänemark gehöre, aber eine größtmögliche oder vollständige Unabhängigkeit anstrebe. Die Insel umfasse 2,16 Millionen Quadratkilometer und sei damit circa sechsmal so groß wie Deutschland, allerdings bei einer Bevölkerung von nur circa 57.000 Einwohnern. Die Grönländer hätten sich schon anlässlich des dänischen Referendums zum EG-Beitritt 1973 mehrheitlich gegen eine Mitgliedschaft ausgesprochen, was allerdings ohne Konsequenzen blieb, da eine Mehrheit in Dänemark für den Beitritt stimmte und die grönländischen Stimmen nicht gesondert gezählt wurden.

1985 sei Grönland dann aus der Europäischen Gemeinschaft ausgetreten. Da die Grönländer 
jedoch die dänische Staatsbürgerschaft behielten, blieben sie EU-Bürger. Grönland sei auch als eines der „Überseeischen Länder und Gebiete" weiterhin mit der Europäischen Union assoziiert. Gegenwärtig bestehe zwischen der Europäischen Union und Grönland eine Partnerschaft, die ein Fischereiabkommen sowie eine jährliche Unterstützung in Höhe von 25 Millionen Euro durch die Europäische Union beinhalte. Auch das Interesse anderer internationaler Akteure an Grönland in seiner Rolle als Anrainerstaat der Arktis sei gestiegen.

\section{Spitzbergen - neutrale Heimat der Polarbären}

Kristian Atland sprach über den Status der Inselgruppe Spitzbergen (Svalbard). Auf Spitzbergen lebten schätzungsweise 2.700 Menschen und 3.000 Polarbären. Die Inseln seien vermutlich zuerst von dem dänischen Entdecker Willem Barentsz im Jahr 1596 entdeckt worden. Vor der Unterzeichnung des Vertrags von Spitzbergen habe man die Inseln primär als Niemandsland betrachtet. Die hauptsächlichen wirtschaftlichen Aktivitäten stellten heute Kohlebergbau, universitäre Forschung sowie Tourismus dar.

Der Vertrag über den Status von Spitzbergen sei 1920 in Paris beschlossen und inzwischen von 42 Staaten unterzeichnet worden. Die wichtigsten Prinzipien stellten die Souveränität Norwegens über Spitzbergen, das Prinzip der Nichtdiskriminierung anderer Staaten bezüglich der Nutzung Spitzbergens, die Erhaltung der natürlichen Umwelt, ein steuerlicher Sonderstatus sowie Einschränkungen für die militärische Nutzung dar.

Bei einem Vergleich zwischen Spitzbergen und Grönland gebe es einige Gemeinsamkeiten, wie die geografische Nähe, den Inselstatus, die geringe Einwohnerzahl, die politische Verbindung zum europäischen Festland sowie die Nichtzugehörigkeit zur Europäischen Union. Bei den Unterschieden falle auf, dass Grönland eine stärkere politische Autonomie aufweise, wie eine eigene Regierung und Flagge. Außerdem verfüge Grönland über eine große indige- ne Bevölkerung (über 80 Prozent der Einwohner), während es in Spitzbergen keine indigenen Bewohner gebe. Schließlich gehöre Spitzbergen geografisch zu Europa und sei demilitarisiert und Grönland gehöre geografisch zu Nordamerika und werde militärisch von Dänemark und den USA genutzt.

Aktuelle Kontroversen bestünden unter anderem über Fischerei- und Bergbaurechte. Norwegen etablierte 1977 eine Schutzzone für Fischereibestände. Seine Küstenwache überwache die Fischerei in dieser Zone gemäß einer nichtdiskriminierenden Praxis, die allen Beteiligten zu Gute komme. Die Verwaltung der Fischereibestände sei erfolgreich, da es große Fischbestände gebe, die nachhaltig bewirtschaftet würden. Allerdings gebe es Konflikte mit Russland. Russische Fischer hielten sich nicht immer an die Quoten. Aus diesem Grund gebe es immer wieder Zwischenfälle unter Beteiligung der norwegischen Küstenwache und russischer Fischer.

Ein weiterer Konflikt betreffe den Festlandsockel (,continental shelf") von Spitzbergen. Die Position der norwegischen Regierung bestehe darin, dass der gleiche Zugang zu natürlichen Ressourcen außerhalb der Zwölf-Meilen-Zone nicht gewährt werden müsse. Andere Vertragsstaaten, wie Russland, Großbritannien, Spanien oder Island, teilten diese Sichtweise nicht. Sie betrachteten die Seeterritorien außerhalb der Zwölf-Meilen-Zone nicht als norwegische Gewässer, sondern als Gewässer, die unter den Vertrag von Spitzbergen fallen und damit allen Vertragsparteien für die Nutzung zur Verfügung stehen müssten. Zurzeit seien die umstrittenen Gewässer nicht für die Ausbeutung natürlicher Ressourcen geöffnet.

Mögliche Lösungen für den Konflikt um die Interpretation des Festlandsockels und der zugehörigen Seeterritorien könnten in der Einberufung einer Konferenz zur erneuten Verhandlung des Status von Spitzbergen bestehen, einem Verfahren vor dem Internationalen Gerichtshof, einer informellen Verhandlungslösung oder, am wahrscheinlichsten, einer Beibehaltung des Status quo. 
Ein weiterer Konflikt bestehe in der Bewertung der Bedeutung des Prinzips des Umweltschutzes im Gegensatz zu den Bergbaurechten. Etwa 65 Prozent von Spitzbergen bestünden aus Naturparks und Schutzgebieten. Russland beispielsweise bewerte in der Regel die Bergbaurechte höher als den Umweltschutz.

Darüber hinaus verdächtige Russland das norwegische Militär, Spitzbergen militärisch zu nutzen. So kritisiere Russland eine norwegische Radarstation auf Spitzbergen. Norwegen weise diesen Verdacht aber zurück und erlaube Russland Inspektionen der entsprechenden Radarstation. Norwegische Kriegsschiffe und Kampfjets dürften aber Spitzbergen besuchen.

Insgesamt werde die norwegische Souveränität über Spitzbergen nicht bezweifelt, aber es gebe Konflikte um die Frage, wie Norwegen Spitzbergen verwalte. Die Zunahme des Interesses an der Arktis und ihrer Ressourcen steigere auch das Interesse an Spitzbergen. Norwegen und Russland hätten im Jahr 2010 einen wichtigen Grenzvertrag zur Festlegung der maritimen Grenze in der Barentssee geschlossen.

Schließlich sollte Norwegen nach Ansicht von Åtland die Interessen aller Parteien des Vertrags von Spitzbergen respektieren und gleichzeitig seine Souveränität über Spitzbergen in einer konsistenten und entschlossenen Weise ausüben.

\section{Nichtanerkannte Kleinstaaten im postsowje- tischen Raum}

Otto Luchterhandt referierte über den völkerrechtlichen Status der, de-facto-Staaten ' in Europa, insbesondere auf dem Gebiet der ehemaligen Sowjetunion. Die, de-facto-Staaten` verfügten über die drei von Georg Jellinek aufgestellten Kriterien für einen Staat: ein Staatsgebiet, ein Staatsvolk und eine unabhängige Staatsgewalt, aber ihnen fehlten die Anerkennung durch die internationale Gemeinschaft. Die ,de-facto-Staaten' seien vollständig unabhängig von den Staaten, zu denen sie ursprünglich gehörten.
Die Sezessionen in den postsowjetischen Staaten hätten sich vor allem durch Unabhängigkeitskriege vollzogen. Die Sezessionen seien meist möglich geworden, weil die Staaten, von denen sich die ,de-facto-Staaten' abspalteten, zu den Zeiten der Sezessionen in interne Machtkonflikte verstrickt gewesen seien.

Abchasien und Berg-Karabach verfügten bereits in der Sowjetunion über einen Autonomiestatus. Dieser sei bestimmten Völkern innerhalb der Sowjetunion gewährt worden, wenn ein ethnisch definiertes Volk sich auf einem Territorium konzentrierte. Die Sowjetunion ließ sich dabei vom Selbstbestimmungsrecht der Völker und vom Prinzip der „ethnischen Territorialautonomie" leiten. Dieses Prinzip sei im Wesentlichen auch in Russland übernommen worden. Ein Problem bei diesem ethnischen Territorialprinzip sei, dass die namensgebende Ethnie in einigen, de-facto-Staaten' nur eine Minderheit darstelle, beispielsweise früher in Abchasien.

Es bestünden vier Möglichkeiten zur Lösung der ungeklärten Statusfrage der, de-facto-Staaten': erstens, eine völkerrechtliche Anerkennung und Aufnahme in die internationale Gemeinschaft; zweitens, eine Rückkehr in den Staat, von dem sich der ,de-facto-Staat' abgespalten habe; drittens, eine Fusion mit dem Schutzstaat, das heißt mit dem Staat, der den ,de-facto-Staat ${ }^{\star}$ protegiere; und viertens, eine Fortführung des Status quo.

Die erste Möglichkeit der weitgehenden völkerrechtlichen Anerkennung könne für alle ,de-facto-Staaten' als unrealistisch angesehen werden. Die zweite Option der Reintegration der ,de-facto-Staaten ' in die Ursprungsstaaten erscheine in wenigen Fällen, wie Transnistrien, möglich. Die dritte Variante der Vereinigung des Sezessionsstaats mit dem Schutzstaat könne man sich in einigen Fällen vorstellen, so von Abchasien oder Südossetien. Im Falle von Südossetien und von Berg-Karabach seien diese Fusionen logisch, weil die Südosseten das gleiche Volk wie die in Russland lebenden Nordosseten darstellten. Gleiches gelte für Berg- 
Karabach, da dort Armenier lebten und damit eine Fusion mit Armenien nach dem Selbstbestimmungsrecht der Völker legitimiert werden könnte.

Eine Lösung der Probleme der, de-facto-Staaten' könne durch eine Einbindung der Ursprungs- und Schutzstaaten in einen gemeinsamen Prozess, beispielsweise im Rahmen der OSZE, erfolgen. Allerdings habe die OSZE hier bislang keine wesentliche Rolle gespielt. Gleiches gelte für den Europarat, der sich ebenfalls nicht an der Lösung der Problematik der ,de-facto-Staaten` beteilige.

Insgesamt könnten die ,de-facto-Staaten' als Sieg des Prinzips der ethnischen Territorialautonomie betrachtet werden. Die, de-factoStaaten' zeichneten sich durch große Unterschiede aus und dies werfe die Frage nach ihrer langfristigen Regierbarkeit auf. Solange die ,de-facto-Staaten" von ihren Schutzstaaten protegiert würden, blieben diese aber lebensfähig. Von den ,de-facto-Staaten' gehe keine Kriegsgefahr aus. Zum Zeitpunkt der Tagung bestand die einzige realistische Möglichkeit für einen Krieg im Falle Aserbaidschans. Dieser Staat könne durch seine militärische Stärke versucht sein, Berg-Karabach durch einen Krieg zurückzugewinnen.

\section{Die drei ,Bräute', die sich nicht trauen - der Europäische Wirtschaftsraum}

Der Vortrag von Barbara Lippert behandelte den Europäischen Wirtschaftsraum (EWR). Der EWR bestehe in erster Linie für diejenigen Staaten, die der Europäischen Union nicht beitreten wollten, aber die Voraussetzungen erfüllen. Norwegen, Island und Liechtenstein bildeten zusammen mit den EU-Staaten den EWR, der damit gegenwärtig 31 Mitglieder habe.

Der EWR könne als Integration ohne Mitgliedschaft bezeichnet werden. Dabei handele es sich im Wesentlichen um Assoziierungs- und Freihandelsabkommen mit der Europäischen Union. Insbesondere Norwegen verfüge über eine sehr enge Anbindung an die Europäische Union. Die Schweiz hingegen habe eine Reihe bilateraler Abkommen mit der Union außerhalb des EWR geschlossen. Die auf diese Art assoziierten Staaten hätten keinen Einfluss auf Entscheidungsprozesse in der Europäischen Union, nur Möglichkeiten der Konsultationen. Die Staaten müssten im Prinzip EU-Recht nachvollziehen und implementieren ohne im Vorhinein auf deren Verabschiedung Einfluss nehmen zu können. Aus demokratietheoretischer Perspektive sei dies problematisch und es stelle sich die Frage, ob dies langfristig so bleiben könne. Außerdem gebe es eine Reihe praktischer Probleme bei der Kooperation mit der Europäischen Union und der Umsetzung von EU-Recht. Zurzeit lägen etwa 500 europäische Rechtsakte vor, die von den Staaten des EWR noch nicht umgesetzt worden seien.

\section{„EU à la carte“}

Die Norm für EU-Beitrittskandidaten bleibe aber die Vollmitgliedschaft und nicht die Mitgliedschaft im EWR. Übergangsregelungen nach einem Beitritt zeichneten sich nur durch temporäre, sektorspezifische und sachlich begrenzte Maßnahmen aus, wie Beschränkungen bei der Personenfreizügigkeit. Dauerhafte Ausnahmen seien nicht vorgesehen. Im Falle eines Beitritts der Türkei könnten möglicherweise dauerhafte Ausnahmen eingeführt werden. Die rote Linie stelle bislang die Vertretung in den Institutionen der Europäischen Union dar: Neumitglieder hätten immer die vollen Stimmrechte in allen EU-Organen erhalten.

Sobald ein Staat Mitglied werde, verfüge er über vielfältige Optionen über eine differenzierte Integration zu verhandeln. So gebe es die Möglichkeit des ,opting-out', das heißt der Nichtbeteiligung an einigen Bereichen der Integration. Es bestehe aber auch die Möglichkeit des ,opting-in', das heißt der Beteiligung von Nicht-EU-Staaten an Bereichen der europäischen Integration, wie dem Schengenraum.

Darüber hinaus gebe es weitere Varianten der differenzierten Integration, wie eine unter- 
schiedliche Geschwindigkeit bei der Erreichung eines gemeinsamen Zieles, beispielsweise bei der Wirtschafts- und Währungsunion. Die zugrundeliegende Idee bestehe in der Garantie einer größeren Flexibilität und Differenzierung mit dem Ziel der Verhinderung von Blockaden, einer effizienteren Politikgestaltung oder einer weiteren Vertiefung. Das Risiko einer ,flexiblen` Europäischen Union bestehe in der zunehmenden Desintegration, falls einzelne Staaten sich immer öfter für Ausnahmeregelungen entscheiden würden. Weitere Probleme bei einer differenzierten Integration lägen in der schwierigeren Ausgestaltung der EU-Institutionen, ihrer Entscheidungsverfahren und ihrer Legitimität.

Perspektivisch könne die Einführung einer EU-Teilmitgliedschaft erwogen werden, wie von Andrew Duff vorgeschlagen. Vorteile bestünden in einer größeren Rechtssicherheit und mehr politischem Spielraum. Ein Nachteil wäre die Gefahr der Desintegration, des ,Europas à la carte'. Weitere Alternativen könnten in der Öffnung von mehr Politikfeldern der Union für Drittstaaten bestehen, der Schaffung eines neuen Status für Drittstaaten oder der Etablierung von „besonderen Beziehungen“.

\section{Die Eurasische Union - Dominanz Russlands}

Hannes Adomeit referierte über die Eurasische Union und ihre Bedeutung. Die Basis für die Eurasische Union bilde die Zollunion zwischen Belarus, Kasachstan und Russland. Die Freizügigkeit von Kapital, Waren, Dienstleistungen und Personen sowie eine koordinierte Wirtschaftspolitik seien weitere Ziele. Darüber hinaus werde die Einbindung von Kirgisistan und Tadschikistan sowie weiterer Staaten angestrebt. Der russische Präsident Wladimir Putin sehe die Eurasische Union dabei nicht als Konkurrenz zur Europäischen Union.
Seit Anfang 2012 gebe es eine Eurasische Wirtschaftskommission mit den drei Ministerpräsidenten und neun Ministern, aus jedem Land drei, die einstimmige Entscheidungen zu Zollbestimmungen, Subventionen oder anderen Regelungen zum gemeinsamen Wirtschaftsraum träfen. Es gebe bereits über tausend Mitarbeiter der Eurasischen Wirtschaftskommission, von denen über 80 Prozent russische Staatsbürger seien. Die Zahl der Mitarbeiter werde anhand der Wirtschaftsleistung der Staaten bemessen.

Russland verstehe Integration vor allem supranational und mit der Zielsetzung der Aufrechterhaltung des russischen Einflusses im postsowjetischen Raum. Die Eurasische Union müsse außerdem primär als politisches Projekt und nicht als wirtschaftliches verstanden werden. So sei Russland bereit, wirtschaftliche Konzessionen zu machen, um dieses Projekt zu unterstützen. Die beiden Staaten Kasachstan und Belarus spielten für die russische Außenwirtschaft kaum eine Rolle (zusammen 7,5 Prozent des russischen Außenhandels). Der wichtigste Handelspartner von Russland sei die Europäische Union, die etwa 50 Prozent der russischen Exporte abnehme. Hingegen sei Russland für Belarus der zentrale Handelspartner mit fast einem Außenhandelsanteil von 50 Prozent.

Das Hauptproblem der Eurasischen Union liege in der autoritären Struktur und der grassierenden Korruption in allen drei beteiligten Staaten (Plätze 123, 127 und 140 im „Transparency Corruption Perception Index“ 2013). ${ }^{2}$ In solch korrupten Staaten könne keine Wirtschaftsunion, die auf fairem Wettbewerb und rechtsstaatlichen Verfahren zur Streitbeilegung beruhen solle, existieren. Es dominiere der mächtigste Staat und dies sei Russland. Entsprechend sieht Adomeit die Zukunft der Eurasischen Union skeptisch.

2 Transparency International: Corruption Perceptions Index 2013, abrufbar unter: http://cpi.transparency.org/ cpi2013/ (letzter Zugriff: 17.3.2014). 